Document downloaded from:

http://hdl.handle.net/10251/180496

This paper must be cited as:

Cantó Colomina, B.; Coll, C.; Sánchez, E. (2009). Structural Identifiability of Linear Singular Systems. Lecture Notes in Control and Information Sciences. 389:243-249. https://doi.org/10.1007/978-3-642-02894-6_23

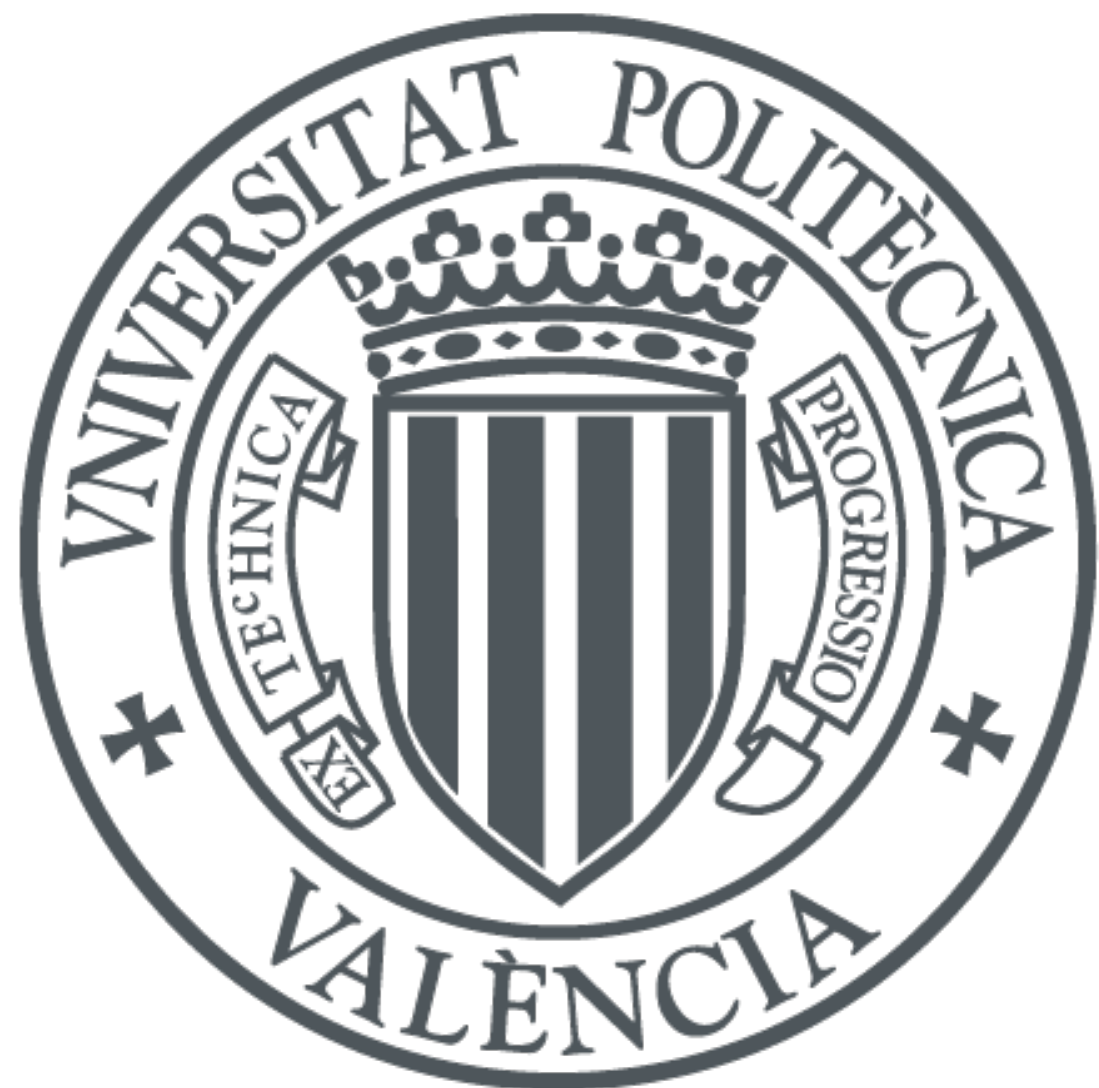

The final publication is available at

https://doi.org/10.1007/978-3-642-02894-6_23

Copyright SPRINGER-VERLAG BERLIN, HEIDELBERGER PLATZ 3, BERLIN, GERMANY, D-14197

Additional Information 


\title{
Structural identifiability of linear singular dynamic systems
}

\author{
Begoña Cantó, Carmen Coll and Elena Sánchez
}

\begin{abstract}
Structured singular systems depending on a parametric vector are considered. The identification of the parameters is analyzed in terms of the input-output behavior of the system. The role of the reachability and observability properties in this analysis is studied and a characterization of the structural identifiability property is given. Finally, the structural identifiability of a positive reachable system is studied.
\end{abstract}

\section{Introduction}

Structured systems are used in the modeled of mechanical, electrical, biological and economics models. Normally, the mathematical model incorporates parameters that symbolize empirical relations among variables. Given a parametrized state space model, structural identifiability is concerned with whether the unknown parameters within the model can be identified from the experiment considered. A structural global identifiability analysis of the model is important in the modeling process and is necessary for the system identification or the parameter estimation. It means uniqueness of the parametric structure in the model. If the structural global identifiability property holds, it is possible to determine the values of the parameters uniquely in terms of known quantities. Several results on structured and global

Begoña Cantó

Institut de Matemàtica Multidisciplinar, Universitat Politècnica de València, 46071 València, Spain, e-mail: bcanto@mat.upv.es

Carmen Coll

Institut de Matemàtica Multidisciplinar, Universitat Politècnica de València, 46071 València, Spain, e-mail: mccoll@mat.upv.es

Elena Sánchez

Institut de Matemàtica Multidisciplinar, Universitat Politècnica de València, 46071 València, Spain, e-mail: esanchezj@mat.upv.es 
identifiability linear system have been published. For instance, a survey on linear structured systems is given in [6] and references therein. For references of global identifiability see [1] and [8]. In the singular case, few results have been given, see for instance [2] and [7]. In this paper a characterization for the structural identifiability of linear singular systems is treated.

The motivation for the study of structural identifiability of positive systems is the used of positive structured linear systems in economics, chemistry and other research areas. In these systems the structure of coefficients matrices plays an important role because there are the positive restrictions on the behavior of the system.

A powerful tool used in the solution of the singular systems is the Drazin inverse. Let $M \in \mathbb{C}^{n \times n}$ a square matrix. The matrix $M^{D}$ is the Drazin inverse of $M$ if it satisfies $M^{D} M M^{D}=M^{D}, M M^{D}=M^{D} M$ and $M^{k+1} M^{D}=M^{k}$, where $k=\operatorname{ind}(M)$, the least nonnegative integer such that $\operatorname{rank}\left(M^{k}\right)=\operatorname{rank}\left(M^{k+1}\right)$, is the index of matrix M.

Consider a structured singular system

$$
\begin{aligned}
E(\mathbf{p}) x(k+1) & =A(\mathbf{p}) x(k)+B(\mathbf{p}) u(k), \\
y(k) & =C(\mathbf{p}) x(k)
\end{aligned}
$$

where the matrices $E(\mathbf{p}), A(\mathbf{p}) \in \mathbb{R}^{n \times n}, B(\mathbf{p}) \in \mathbb{R}^{n \times m}$ and $C(\mathbf{p}) \in \mathbb{R}^{m \times n}$ have a fixed structure with $\mathbf{p}$ belongs to a subset $\mathscr{P} \subseteq \mathbb{R}^{\nabla}$. Matrix $E(\mathbf{p})$ is singular with $n_{1}=$ $\operatorname{rank}\left(E^{D}(\mathbf{p}) E(\mathbf{p})\right)$ and $l=\operatorname{ind}(E(\mathbf{p}))$. This structured singular system is denoted by $S(\mathbf{p})=(E(\mathbf{p}), A(\mathbf{p}), B(\mathbf{p}), C(\mathbf{p}), \mathscr{P})$. If there exists $\lambda \in \mathbb{C}$ such that $\operatorname{det}[\lambda E(\mathbf{p})-$ $A(\mathbf{p})] \neq 0$ the system $S(\mathbf{p})$ has solution. The output of the system is given by

$$
\begin{aligned}
y(k)= & \left(C(\mathbf{p})\left(E^{D}(\mathbf{p}) A(\mathbf{p})\right)^{k} E^{D}(\mathbf{p}) E(\mathbf{p}) x(0)\right. \\
& +\sum_{i=0}^{k-1} C(\mathbf{p}) E^{D}(\mathbf{p})\left(E^{D}(\mathbf{p}) A(\mathbf{p})\right)^{k-i-1} B(\mathbf{p}) u(i) \\
& -C(\mathbf{p})\left(I-E^{D}(\mathbf{p}) E(\mathbf{p})\right) \sum_{i=0}^{l-1}\left(E(\mathbf{p}) A^{D}(\mathbf{p})\right)^{i} A^{D}(\mathbf{p}) B(\mathbf{p}) u(k+i),
\end{aligned}
$$

if $E(\mathbf{p}) A(\mathbf{p})=A(\mathbf{p}) E(\mathbf{p})$ and $\operatorname{ker}(E(\mathbf{p})) \cap \operatorname{ker}(A(\mathbf{p}))=\{0\}$. The admissible initial conditions set, $\mathscr{X}_{0}$, is given by

$$
\mathscr{X}_{0}=\operatorname{Im}\left[E^{D}(\mathbf{p}) E(\mathbf{p}) H_{0} \ldots H_{q-1}\right]
$$

where $H_{i}=\left(I-E^{D}(\mathbf{p}) E(\mathbf{p})\right)\left(E(\mathbf{p}) A^{D}(\mathbf{p})\right)^{i} A^{D}(\mathbf{p}), i=0, \ldots, l-1$.

Before solving the identifiability problem we will remind the definition of reachable, observable and similar properties.

A system $S(\mathbf{p})$ is called structurally reachable if, for all $\mathbf{p} \in \mathscr{P}$ the reachability matrices 


$$
\begin{aligned}
\mathscr{R}_{f}(S(\mathbf{p}))= & {\left[E^{D}(\mathbf{p}) B(\mathbf{p}) \ldots E^{D}(\mathbf{p})\left(E^{D}(\mathbf{p}) A(\mathbf{p})\right)^{n-1} B(\mathbf{p})\right] } \\
\mathscr{R}_{b}(S(\mathbf{p}))= & {\left[\left(I-E^{D}(\mathbf{p}) E(\mathbf{p})\right) A^{D}(\mathbf{p}) B(\mathbf{p}) \ldots\right.} \\
& \left.\ldots\left(I-E^{D}(\mathbf{p}) E(\mathbf{p})\right)\left(E(\mathbf{p}) A^{D}(\mathbf{p})\right)^{l-1} A^{D}(\mathbf{p}) B(\mathbf{p})\right],
\end{aligned}
$$

have full rank, that is $\operatorname{rank}\left(\mathscr{R}_{f}(S(\mathbf{p}))\right)=n_{1}$ and $\operatorname{rank}\left(\mathscr{R}_{b}(S(\mathbf{p}))\right)=n-n_{1}$. And, a system $S(\mathbf{p})$ is called structurally observable if, for all $\mathbf{p} \in \mathscr{P}$ the observability matrices

$$
\begin{aligned}
\mathscr{O}_{f}(S(\mathbf{p}))= & {\left[\left(C(\mathbf{p}) E^{D}(\mathbf{p})\right)^{T} \ldots\left(C(\mathbf{p}) E^{D}(\mathbf{p})\left(E^{D}(\mathbf{p}) A(\mathbf{p})\right)^{n-1}\right)^{T}\right]^{T} } \\
\mathscr{O}_{b}(S(\mathbf{p}))= & {\left[\left(C(\mathbf{p})\left(I-E(\mathbf{p})^{D} E(\mathbf{p})\right) A^{D}(\mathbf{p})\right)^{T} \ldots\right.} \\
& \left.\ldots\left(C(\mathbf{p})\left(I-E^{D}(\mathbf{p}) E(\mathbf{p})\right)\left(E(\mathbf{p}) A^{D}(\mathbf{p})\right)^{l-1} A^{D}(\mathbf{p})\right)^{T}\right]^{T}
\end{aligned}
$$

have full rank, that is $\operatorname{rank}\left(\mathscr{O}_{f}(S(\mathbf{p}))\right)=n_{1}$ and $\operatorname{rank}\left(\mathscr{O}_{b}(S(\mathbf{p}))\right)=n-n_{1}$.

Two systems $S(\mathbf{p})$ and $S(\mathbf{q}), \mathbf{p}, \mathbf{q} \in \mathscr{P}$ are structurally similar if there exists an invertible matrix $T$ such that $E(\mathbf{p})=T E(\mathbf{q}) T^{-1}, A(\mathbf{p})=T A(\mathbf{q}) T^{-1}, B(\mathbf{p})=T B(\mathbf{q})$ and $C(\mathbf{p})=C(\mathbf{q}) T^{-1}$.

\section{Structural identifiability problem}

The identifiability of the parameters of the system is concerned with the determination of these from the external behavior of the system. The response of the system from an input given can be analyzed in the z-domain or using the input-output application. That is, to determine the input-output behavior (io) of a model $S(\mathbf{p})$ we can use the transfer matrix

$$
G(z, \mathbf{p})=C(\mathbf{p})(z E(\mathbf{p})-A(\mathbf{p}))^{-1} B(\mathbf{p})
$$

or the Markov parameters associated to the system $S(\mathbf{p})$. These parameters are given by

$$
\begin{aligned}
V(j, \mathbf{p}) & =C(\mathbf{p}) E^{D}(\mathbf{p})\left(E^{D}(\mathbf{p}) A(\mathbf{p})\right)^{j} B(\mathbf{p}), j \geq 0 \\
H(j, \mathbf{p}) & =C(\mathbf{p})\left(I-E(\mathbf{p}) E^{D}(\mathbf{p})\right)\left(E(\mathbf{p}) A^{D}(\mathbf{p})\right)^{j} A^{D}(\mathbf{p}) B(\mathbf{p}) \\
j & =0, \ldots, l-1 .
\end{aligned}
$$

The concept of structural identifiability is given in the following definition.

Definition 1. The system $S(\mathbf{p})$ is structurally identifiable if and only if, for almost any two candidates parameter vector values $\mathbf{p}, \mathbf{q} \in \mathscr{P}$, io $(\mathbf{p})=i o(\mathbf{q})$ implies $\mathbf{p}=\mathbf{q}$, where io $(\cdot)$ denotes the input-output behavior of the system $S(\mathbf{p})$.

This concept is also named in the literature global identifiability (see [2], [8] to continuous-time case). The structural reachability and observability are related to 
the structural identifiability property. However, these properties are neither necessary nor sufficient conditions for global identifiability. For example, consider the system $S(\mathbf{p})$ with the following structure

$$
E(\mathbf{p})=\left(\begin{array}{rrrr}
p_{1} & p_{2} & 0 & 0 \\
0 & p_{3} & 0 & 0 \\
0 & 0 & 0 & p_{4} \\
0 & 0 & 0 & 0
\end{array}\right), A(\mathbf{p})=I+E(\mathbf{p}), B=\left(\begin{array}{ll}
0 & 1 \\
1 & 0 \\
0 & 0 \\
1 & 1
\end{array}\right),
$$

and

$$
C=\left(\begin{array}{llll}
1 & 0 & 1 & 0 \\
0 & 1 & 0 & 1
\end{array}\right)
$$

where the parametric vector $\mathbf{p} \in \mathscr{P}=\left\{\left(p_{1}, p_{2}, p_{3}, p_{4}\right) \in \mathbb{R}_{+}^{4}, p_{i}>0\right\}$. Constructing the reachability and observability matrices it is easy to see that they have full rank, then the system is structurally reachable and observable but the system is unidentifiable since its transfer matrix is

$$
G(z, \mathbf{p})=\left(\begin{array}{rr}
\frac{-p_{2}(z-1)}{\left(z p_{1}-1-p_{1}\right)\left(z p_{3}-1-p_{3}\right)} & p_{4}(1-z) \\
\frac{1}{z p_{3}-1-p_{3}} & -1
\end{array}\right)
$$

and the parameters are undetermined. Then they cannot be estimated using inputoutput data, even using well-design experiments.

On the other hand there exist structurally globally identifiable systems which are not structurally reachable. For example, the structured system $(A(\mathbf{p}), B)$ given by

$$
A(\mathbf{p})=\left(\begin{array}{rr}
1-\alpha & 0 \\
0 & 1-\alpha
\end{array}\right), B=\left(\begin{array}{l}
1 \\
0
\end{array}\right)
$$

with the parametric vector $\mathbf{p} \in\{\alpha \in \mathbb{R} / \alpha \neq 0\}$. Since the reachability matrix satisfies

$$
\operatorname{rank}\left(\begin{array}{rr}
1 & 1-\alpha \\
0 & 0
\end{array}\right)=1
$$

the system is not reachable but as its transfer function is

$$
G(z, \mathbf{p})=\frac{1}{z+\alpha-1}\left(\begin{array}{l}
1 \\
0
\end{array}\right),
$$

the parameter $\alpha$ is uniquely determined and the system is globally identifiable.

In the following result we have a characterization of the structural global identifiability property when the system is structurally reachable and observable.

Theorem 1. Consider the structured system $S(\boldsymbol{p})$ given in (1) structurally reachable and structurally observable. Then,

$S(\boldsymbol{p})$ is structurally identifiable if and only if $S(\boldsymbol{p})$ and $S(\boldsymbol{q})$ structurally similar implies that $\boldsymbol{p}=\boldsymbol{q}$ and $T=I$. 
Proof. Consider $S(\mathbf{p})$ and $S(\mathbf{q})$ structurally similar, then they have the same Markov parameters. That is, the input-output behavior is the same $i o(\mathbf{p})=i o(\mathbf{q})$ and by hypothesis $\mathbf{p}=\mathbf{q}$. Reciprocally, consider $S(\mathbf{p})$ and $S(\mathbf{q})$ such that they have the same input-output behavior. It is known that each one of them is equivalent to (see [5])

$$
\begin{aligned}
& \bar{S}(\mathbf{p})=\left(\left(\begin{array}{cc}
I & O \\
O & N(\mathbf{p})
\end{array}\right),\left(\begin{array}{cc}
A_{1}(\mathbf{p}) & O \\
O & I
\end{array}\right),\left(\begin{array}{l}
B_{1}(\mathbf{p}) \\
B_{2}(\mathbf{p})
\end{array}\right),\left(C_{1}(\mathbf{p}) C_{2}(\mathbf{p})\right)\right) \\
& \bar{S}(\mathbf{q})=\left(\left(\begin{array}{cc}
I & O \\
O & N(\mathbf{q})
\end{array}\right),\left(\begin{array}{cc}
A_{1}(\mathbf{q}) & O \\
O & I
\end{array}\right),\left(\begin{array}{l}
B_{1}(\mathbf{q}) \\
B_{2}(\mathbf{q})
\end{array}\right),\left(C_{1}(\mathbf{q}) C_{2}(\mathbf{q})\right)\right)
\end{aligned}
$$

and they satisfy $i o(\mathbf{p})=i o(\mathbf{q})$. Using the definition (2) of the Markov parameters and the definition of the reachability and observability matrices we show that

$$
\begin{aligned}
\mathscr{O}_{f}(S(\mathbf{p})) B_{1}(\mathbf{p}) & =\mathscr{O}_{f}(S(\mathbf{q})) B_{1}(\mathbf{q}) \\
C_{1}(\mathbf{p}) \mathscr{R}_{f}(S(\mathbf{p})) & =C_{1}(\mathbf{q}) \mathscr{R}_{f}(S(\mathbf{q})) \\
\mathscr{O}_{f}(S(\mathbf{p})) A_{1}(\mathbf{p}) \mathscr{R}_{f}(S(\mathbf{p})) & =\mathscr{O}_{f}(S(\mathbf{q})) A_{1}(\mathbf{q}) \mathscr{R}_{f}(S(\mathbf{q}))
\end{aligned}
$$

and

$$
\begin{aligned}
\mathscr{O}_{b}(S(\mathbf{p})) B_{2}(\mathbf{p}) & =\mathscr{O}_{b}(S(\mathbf{q})) B_{2}(\mathbf{q}) \\
C_{2}(\mathbf{p}) \mathscr{R}_{b}(S(\mathbf{p})) & =C_{2}(\mathbf{q}) \mathscr{R}_{b}(S(\mathbf{q})) \\
\mathscr{O}_{b}(S(\mathbf{p})) N(\mathbf{p}) \mathscr{R}_{b}(S(\mathbf{p})) & =\mathscr{O}_{b}(S(\mathbf{q})) N(\mathbf{q}) \mathscr{R}_{b}(S(\mathbf{q})) .
\end{aligned}
$$

Constructing $T=\left(\begin{array}{cc}T_{f} & O \\ O & T_{b}\end{array}\right)$ with

$$
\begin{gathered}
T_{f}=\mathscr{R}_{f}(S(\mathbf{p})) \mathscr{R}_{f}^{T}(S(\mathbf{q}))\left(\mathscr{R}_{f}(S(\mathbf{q})) \mathscr{R}_{f}^{T}(S(\mathbf{q}))\right)^{T} \\
T_{b}=\mathscr{R}_{b}(S(\mathbf{p})) \mathscr{R}_{b}^{T}(S(\mathbf{q}))\left(\mathscr{R}_{b}(S(\mathbf{q})) \mathscr{R}_{b}^{T}(S(\mathbf{q}))\right)^{T}
\end{gathered}
$$

and using (4)-(5) it is easy to show that $T$ is nonsingular and the systems $\bar{S}(\mathbf{p})$ and $\bar{S}(\mathbf{q})$ are similar under the transformation matrix $T$. By hypothesis, this implies that $T=I$ and $\mathbf{p}=\mathbf{q}$, and hence, the structured system is identifiable.

Before we have indicated that the structured system $S(\mathbf{p})$ given by (3) is unidentifiable, now we can observe that it neither satisfies the condition of the theorem 1. Consider two structured systems $S(\mathbf{p})$ and $S(\mathbf{q})$ of type (3) such that

$$
E(\mathbf{p})=\left(\begin{array}{rrrr}
p_{1} & p_{2} & 0 & 0 \\
0 & p_{3} & 0 & 0 \\
0 & 0 & 0 & p_{4} \\
0 & 0 & 0 & 0
\end{array}\right) \text { and } E(\mathbf{q})=\left(\begin{array}{rrrr}
p_{1} & p_{2} & 0 & 0 \\
0 & p_{3} & 0 & 0 \\
0 & 0 & 0 & q_{4} \\
0 & 0 & 0 & 0
\end{array}\right)
$$

We can prove that these systems are similar by means of the transformation matrix 


$$
L=\left(\begin{array}{rrrr}
1 & 0 & 0 & 0 \\
0 & 1 & 0 & 0 \\
\frac{p_{4}}{q_{4}} & 0 & 1 & 0 \\
0 & 0 & 0 & 1
\end{array}\right) \text {. }
$$

Hence, if we choose $p_{4} \neq q_{4}$ then $\mathbf{p}=\left(p_{1}, p_{2}, p_{3}, p_{4}\right) \neq\left(p_{1}, q_{2}, p_{3}, q_{4}\right)=\mathbf{q}$ and $L \neq I$.

\section{Structural identifiability of positive systems}

When positive restrictions are considered a concept of reachability of positive states by means of positive controls is used. This concept is named positive reachability (see [3]). In this context, we are interested in the study of the structural identifiability problem when the system satisfies this property. Thus, we say that a structured system $S(\mathbf{p})$ is

(i) structurally identifiable positive system if it is positive for all $\mathbf{p} \in \mathscr{P}$ and it is structurally identifiable, and

(ii) structurally reachable positive system if it is positive and positively reachable for all $\mathbf{p} \in \mathscr{P}$.

Consider the system $S(\mathbf{p})$ where the matrices are given by

$$
E=\left(\begin{array}{cc}
D & O \\
O & N
\end{array}\right) \geq 0, A(\mathbf{p})=\left(\begin{array}{cc}
A_{1}(\mathbf{p}) & O \\
O & I
\end{array}\right) B(\mathbf{p})=\left(\begin{array}{c}
B_{1}(\mathbf{p}) \\
B_{2}
\end{array}\right)
$$

where $D$ is a nonsingular diagonal matrix, $N$ is a nilpotent matrix, $B_{2} \leq 0$, and

$$
A_{1}(\mathbf{p})=\left(\begin{array}{cccc}
0 & p_{1} & 0 & 0 \\
0 & 0 & \ddots & 0 \\
0 & \ddots & \ddots & p_{n_{1}-1} \\
p_{n_{1}} & 0 & 0 & 0
\end{array}\right) \text {, and } B_{1}(\mathbf{p})=\left(\begin{array}{c}
0 \\
\vdots \\
0 \\
b
\end{array}\right) \text {, }
$$

being $\mathscr{P}=\left\{\mathbf{p}=\left(p_{1}, \ldots, p_{n_{1}}, b\right) \in \mathbb{R}^{n_{1}+1} / p_{i}, b>0\right\}$. This structured system is positive since it holds the conditions established in [4] in order to be a positive system, that is, $E^{D} E \geq 0, E^{D} A(\mathbf{p}) \geq 0, E^{D} B(\mathbf{p}) \geq 0$, and $\left(I-E^{D} E\right)\left(E A^{D}(\mathbf{p})\right)^{i} A^{D}(\mathbf{p}) B(\mathbf{p}) \leq$ $0, i=0,1, \ldots, l-1$, where $l$ is the index of $E$. Moreover, the reachability matrices $\mathscr{R}_{f}(S(\mathbf{p}))$ and $\mathscr{R}_{f}(S(\mathbf{p}))$ have a monomial matrix of size $n_{1}$ and $n-n_{1}$, respectively, then the positive structural reachability property holds (see [3]).

In the following result we prove that it is also structurally globally identifiable.

Theorem 2. Consider the positive structured system (6). This system is structurally globally identifiable. 
Proof. We consider two positive structured systems $S(\mathbf{p})$ and $S(\mathbf{q})$ of type (6) with $\mathbf{p}, \mathbf{q} \in \mathscr{P}$ such that they have the same input-output behavior (io)

$$
\begin{aligned}
& V(j, \mathbf{p})=V(j, \mathbf{q}), j \geq 0 \\
& H(j, \mathbf{p})=H(j, \mathbf{q}), j=0, \ldots, l-1,
\end{aligned}
$$

and we shall prove that $\mathbf{p}=\mathbf{q}$. If $\mathbf{p}=\left(p_{1}, \ldots, p_{n 1}, b\right)$ and $\mathbf{q}=\left(q_{1}, \ldots, q_{n 1}, \bar{b}\right)$, by definition of the Markov parameters in (2) and by the structure of the matrices of the system (6) we have

$$
\begin{aligned}
E^{D} B(\mathbf{p}) & =E^{D} B(\mathbf{q}) \Rightarrow b=\bar{b} \\
E^{D} A(\mathbf{p}) B(\mathbf{p}) & =E^{D} A(\mathbf{q}) B(\mathbf{q}) \Rightarrow p_{n_{1}-1}=q_{n_{1}-1} \\
& \vdots \\
\left(E^{D} A(\mathbf{p})\right)^{n_{1}-1} B(\mathbf{p}) & =\left(E^{D} A(\mathbf{q})\right)^{n_{1}-1} B(\mathbf{q}) \Rightarrow p_{1}=q_{1} \\
\left(E^{D} A(\mathbf{p})\right)^{n_{1}} B(\mathbf{p}) & =\left(E^{D} A(\mathbf{q})\right)^{n_{1}} B(\mathbf{q}) \Rightarrow p_{n_{1}}=q_{n_{1}}
\end{aligned}
$$

Hence, $\mathbf{p}=\mathbf{q}$.

\section{Conclusions}

The problem of structural global identifiability of the model consists in determinate the uniqueness of the parameter vector when an input-output response is considered. This problem has been studied for structured singular systems. The relation between the structural properties and the structural identifiability has been analyzed for these systems and a characterization of this property has been given. Finally, the structural identifiability of a class of positive reachable system has been treated.

Acknowledgements Supported by Grant MMT2007-64477.

\section{References}

1. Audoly, S., D'Angió, L., Saccomani, M.P., Cobelli, C.: Global identifiability of linear compartmental models. IEEE Trans. Biomed. Eng. 45, 36-47 (1998)

2. Ben-Zvi, A., McLellan, P.J., McAuley, K.B.: Identifiability of linear time-invariant differential-algebraic systems. I. The generalized Markov parameter approach. Ind. Eng. Chem. Res. 42, 6607-6618 (2003)

3. Bru, R., Coll, C., Romero, S., Sánchez, E.: Some problems about structural properties of positive descriptor systems. In: Benvenuti, L., De Santis, A., Farina, L. (eds.) Lecture Notes in Control and Information, pp. 233-240. Springer, Heidelberg (2003) 
4. Bru, R., Coll, C., Sánchez, E.: Structural properties of positive linear time-invariant difference-algebraic equations. Linear Algebra and its Applications. 349, 1-10 (2002)

5. Dai, L.: Singular Control Systems. In: Thoma, M., Wyner, A. (eds.) Lecture Notes in Control and Information Sciences, book. Springer, Heidelberg (1989)

6. Dion, J.M., Commault, C., Van der Woude, J.: Generic properties and control of linear structured systems: a survey. Automatica. 39, 1125-1144 (2003)

7. Miyamura, A., Kazuyuki, K.: Identifiability of delayed singular systems. In Proceedings 5th Asian Control Conference, pp. 789-797. Melbourne, Australia (2004)

8. Tayakout-Fayolle, M., Jolimaitre, E., Jallut, C.: Consequence of strutural identifiability properties on state model formulation for linear inverse chromatography. Chemical Eng. Science 55, 2945-2956 (2000) 